\title{
A NOVEL SLIDING MODE CONTROLLER FOR FUNCTIONAL ELECTRICAL STIMULATION
}

\author{
Sašo Jezernik*, Philipp Inderbitzin*, Thierry Keller*, Robert Riener ${ }^{* *}$ \\ " ETH Zürich, Automatic Control Laboratory, Physikstrasse 3, 8092 Zürich, Switzerland \\ ** Technical University of Munich, Inst. of Automatic Control Eng., Arcisstrasse 21, 80333 Munich, Germany
}

\begin{abstract}
This article describes a model-based development of a new nonlinear controller for control of Functional Electrical Stimulation, which can be used to restore movement in paralyzed individuals. The control design is based on the theory of sliding mode control. The controller is mathematically derived and shown to provide asymptotic stability of knee joint angle tracking by electrical stimulation of knee extensor muscle group only, or by electrical stimulation of knee extensor and flexor muscle groups. Its behaviour was evaluated in simulations with artificial and physiological knee joint angle reference trajectories. The controller was able to track trajectories with a period of $2 \mathrm{~s}$ with a root-mean-square error of approximately 2 degrees, which is considered a good performance. It was also shown to be robust to parameter variations of the model. This is important as models for different persons will differ considerably. Copyright (C) 2002 IFAC
\end{abstract}

Keywords: applied neural control, medical applications, biomechanics

\section{FUNCTIONAL ELECTRICAL STIMULATION}

Electrical current pulses delivered via electrodes located in the vicinity of nerve fibers can be used to depolarize nerve fiber's membrane potential. Provided that the depolarization is strong enough, this leads to a generation of an action potential that is propagated along the nerve fiber. In case of a motor nerve fiber the action potential will propagate to the innervated muscle and will cause a muscle contraction. This muscle contraction in turn generates an active muscle force that creates a corresponding active joint torque producing a body movement (force is transmitted to the bones via muscle tendons).

Normally the action potentials are generated by the nervous activity of the brain and the spinal cord. However, in some cases of dysfunction - like for example in individuals with paralysis or some other types of neuropathies - the action potential generation or propagation is impaired or not possible. In such cases the action potentials may be generated artificially as described above. If the stimulation is furthermore used to restore some lost physiological function it is termed Functional Electrical Stimulation (FES).

In this work we are concerned with the application of FES to restore movements of the lower limbs and moreover with the development of stable and robust closed-loop control techniques to control these movements. So far the open-loop controlled electrical stimulation techniques have been predominantly used, because of the complexity of the system (nonlinearities, time-variations, limited actuator (muscle) band-width and power) (Crago, et al., 1996). Many researchers (including ourselves) however are convinced that modern control theory techniques could improve the performance and accuracy of the artificially generated movement, although the situations with the need of better performance have to be discussed and carefully selected.

The contribution of this article is a model-based development of a sliding mode controller to control the movement of the lower leg. This is a continuation of our earlier work reported in (Jezernik and Riener, 1999). More details about the sliding mode control can be, for example, found in (Utkin, 1992) and (Slotine and Coetsee, 1986). An asymptotically stable controller has been designed that is able to track fast (physiological) movements of the knee joint via FES of the corresponding knee joint extensor and flexor muscles. The controller has been tested in computer simulations. It achieved good knee angle tracking and was also shown to be robust to various parameter variations.

In the next section we will describe the model used in the controller design, in the third section the actual controller, in the fourth section we will show the results, and in the last section we will conclude with a discussion.

\section{PHYSIOLOGICAL AND BIOMECHANICAL MUSCLE-KNEE MODEL}

The human muscle-knee model has been developed in SIMULINK in earlier studies and was also 
validated in actual human experiments (Riener and Fuhr, 1998). It simulates a one degree of freedom movement at the knee joint of a human leg and consists of five muscle group models and a biomechanical knee model part. Each muscle group model consists of a static recruitment block (saturation limits: 50-500 $\mu \mathrm{s}$ ), linear second order Calcium $(\mathrm{Ca})$ release and reuptake model, muscle fatigue block, nervous delay block (25 ms delay), and active muscle force model (Hill-type, with nonlinear force-length and force-velocity relationships). In this study only two muscle group models were used: (a) quadriceps muscle group (knee extensors, with $T=0.05 \mathrm{~s}$ as Ca dynamics time constant), and (b), biceps femoris muscle group (knee flexors, with $T=0.1 \mathrm{~s}$ as $\mathrm{Ca}$ dynamics time constant). The generated muscle forces were multiplied with the corresponding moment arms to calculate the active muscle knee-joint torques, and were fed into the equation of motion. The latter is a nonlinear dynamical equation of second order and comprises total inertia around the knee joint, gravity, linear viscous damping $(d=0.6)$, and is also augmented with the passive knee joint torques modelled as a double exponential function.

The overall model with one muscle group has the following state-space representation:

$$
\left[\begin{array}{c}
\dot{x}_{1}=x_{2} \\
\dot{x}_{2}=\frac{m a\left(x_{1}\right) \cdot f i t \cdot x_{3} \cdot g_{1}\left(x_{1}\right) \cdot g_{2}\left(x_{1}, x_{2}\right)+\tau_{\text {gravity }}\left(x_{1}\right)+\tau_{\text {passive }}\left(x_{1}\right)-d \cdot x_{2}}{I_{\text {tot }}} \\
\dot{x}_{3}=x_{4} \\
\dot{x}_{4}=\frac{1}{T^{2}} \cdot\left(-2 T \cdot x_{4}-x_{3}\right)+\frac{1}{T^{2}} u\left(t-t_{d}\right)
\end{array}\right]
$$

whereby the state space variables $x_{1}-x_{4}$ stand for the knee angle, knee angular velocity, concentration of the $\mathrm{Ca}$ ions, and the derivative of the $\mathrm{Ca}$ ion concentration respectively. In this set of differential equations, $m a$ stands for the muscle moment arm, fit describes the muscle fitness, $g_{1}$ and $g_{2}$ stand for the force-length and force-velocity relationships, $\tau_{g}$ for gravity, $\tau_{p}$ for passive knee joint torques, $d$ for linear damping, $T$ is the Ca release-reuptake time constant, and $t_{d}$ is the delay. $u$ is the input to the model (pulsewidth of the current pulses at stimulation frequency of $20 \mathrm{~Hz}$ ).

Note that this model can be split into two subsystems in the following way:

$$
\begin{aligned}
& \text { Subsystem 1 }\left[\begin{array}{c}
\dot{x}_{1}=f_{1}\left(x_{2}\right) \\
\dot{x}_{2}=f_{2}\left(x_{1}, x_{2}, x_{3}\right)
\end{array}\right] \\
& \text { Subsystem 2 }\left[\begin{array}{c}
\dot{x}_{3}=f_{3}\left(x_{4}\right) \\
\dot{x}_{4}=f_{4}\left(x_{3}, x_{4}, u\right)
\end{array}\right]
\end{aligned}
$$

The state variable $x_{3}$ can now be regarded as an input to the subsystem 1 and as an output of the subsystem 2 , which is driven by the input $u$. It is important to note that $x_{1}$ and $x_{2}$ can be measured, but $x_{3}$ and $x_{4}$ can not.

The above state-space representation becomes in the case of additional muscle groups augmented with additional differential equations for the corresponding $\mathrm{Ca}$ dynamics of each muscle group, and the second differential equation then contains additional active muscle torque terms $m a\left(x_{l}\right) \cdot f i t \cdot g_{1}$ $\left(x_{1}\right) \cdot g_{2}\left(x_{1}, x_{2}\right)$.

\section{SLIDING MODE FES CONTROLLER}

The design of the FES controller was influenced by the specific structure of the model (comprising two subsystems). In a first step, an asymptotically stable sliding mode (SM) controller was designed for the first subsystem only. The sliding surface was selected as:

$$
s(x)=x_{2}+\lambda x_{1}=\dot{x}_{1}+\lambda x_{1}
$$

which then yielded the following control law for $x_{3}$ :

$$
u_{S M_{-} S S 1}=\left(x_{3}\right)=u_{e q}-\hat{k} \cdot \operatorname{sgn}(s)=u_{e q}-\hat{k} \cdot \operatorname{sgn}\left(x_{2}+\lambda \cdot x_{1}\right)=
$$$$
=\frac{1}{m a\left(x_{1}\right) \cdot f i t \cdot g_{1}\left(x_{1}\right) \cdot g_{2}\left(x_{1}, x_{2}\right)}\left[-\lambda \cdot x_{2} \cdot I_{t o t}-\tau_{g}-\tau p+d \cdot x_{2}-I \text { tot } k \cdot \operatorname{sgn}\left(x_{2}+\lambda \cdot x_{1}\right)\right]
$$

It can be easily verified that this discontinuous control law achieves asymptotical stability of knee joint angle and velocity $\left(x_{1} \rightarrow 0\right.$ and $\left.x_{2} \rightarrow 0\right)$. If the term $k \cdot \operatorname{sign}(s)$ is replaced with the term $k \cdot s$ in the above control law, then it is called a continuous sliding mode control law. Both variants, discontinuous and continuous, were evaluated in our study, but in this paper we will report only on the results of the continuous SM controller, which is able to reduce chattering that is usually observed with the discontinuous SM control law (Curk, et al., 1996).

The SM control law was tested directly on subsystem 1 with an additional $\mathrm{ZOH}$ block $(\mathrm{T}=1 / 20 \mathrm{~s}$, because of $20 \mathrm{~Hz}$ stimulation frequency), delay of $25 \mathrm{~ms}$, and saturation levels set to 0 and 1 . It was also tested in combination with the following feed-forward control law for the overall system (again with an additional $\mathrm{ZOH}$ block $(\mathrm{T}=1 / 20 \mathrm{~s}))$ :

$$
u(t)=\frac{\left(2 \cdot T_{t} \cdot s+1\right) \cdot 400}{\left(T_{f} \cdot s+1\right)} \cdot u_{S M_{-} S S 1}+100
$$

with $T_{t}$ being equal to the corresponding Ca releasereuptake time constant, and $\mathrm{T}_{\mathrm{f}}$ being the first order low-pass filter constant equal to 0.01 .

Simulations were first performed with the stimulation of a knee extensor muscle group only. Knee extensors have thereby generated active muscle torque that acted against the gravity and caused lifting of the lower leg. The leg was lowered only passively due to gravity. Finally, the simulations were also performed with the concurrent stimulation of the knee extensors and flexors, whereby active knee flexor torque assisted the gravity in lowering the leg. To do so, the control law was split into positive and negative components accordingly, and subsequently applied to the corresponding muscle groups. 


\section{SIMULATION RESULTS}

We have tracked two kinds of trajectories: (1) ramps with the amplitude of 60 degrees and period of $2 \mathrm{~s}$, and (2), real knee joint angle walking trajectories with the period of $2 \mathrm{~s}$. This period corresponds to a physiological walking speed of approximately 1.9 $\mathrm{km} / \mathrm{h}$. The simulations were performed at different values of controller parameters $\lambda$ and $k$ in order to evaluate their effect on the tracking error. Furthermore, estimates of the model terms fit, $I_{t o t}, \tau_{g}$, and $\tau_{p}$ used in the SM control law were varied by $-50,-20,20$, and $50 \%$, and the robustness of the controller to these parameter variations was examined.

\subsection{Tracking performance}

Table 1 shows the root-mean-square (RMS) tracking error (calculated over one period) as a function of controller parameters $\lambda$ and $k$ when only the knee extensors were stimulated and with correct, nominal controller parameters. The best performance for the examined values is achieved for $\lambda=20$ and $k=0.05$. Theoretically should larger $\lambda$ values accelerate the convergence along the sliding surface to the zero point in the phase-plane (with $1 / \lambda$ being the time constant of $x_{1} \rightarrow 0$ ), and larger $k$ values should accelerate reaching of the sliding surface and provide better robustness and better stability (see below).

Table 1: SM controller for subsystem 1 (one muscle)

\begin{tabular}{c|ccc}
\hline $\mathrm{K}$ & $\begin{array}{c}\mathrm{e}_{\mathrm{RMS}}[\mathrm{deg}] \\
(\lambda=10)\end{array}$ & $\begin{array}{c}\mathrm{e}_{\mathrm{RMS}}[\mathrm{deg}] \\
(\lambda=20)\end{array}$ & $\begin{array}{c}\mathrm{e}_{\mathrm{RMS}}[\mathrm{deg}] \\
(\lambda=30)\end{array}$ \\
\hline 0.01 & 4.81 & 3.79 & 3.47 \\
0.05 & 2.96 & $\mathbf{2 . 9 2}$ & 3.71 \\
0.1 & 2.98 & 4.77 & 5.72 \\
0.2 & 5.56 & 6.65 & 12.98 \\
\hline
\end{tabular}

Fig.1 shows tracking of a ramp trajectory by continuous sliding mode control of a model with one muscle only. The first panel shows the control signal (normalized $\mathrm{Ca}$ ion concentration, $\mathrm{u}_{\mathrm{SM}-\mathrm{SS} 1}$ ), the second panel the reference (solid line) and actual (dashed line) knee joint angle, and the third panel the convergence of the phase trajectory to the sliding surface and to the origin of the phase plane.

Fig. 2 depicts the performance of the combined controller with stimulation of one muscle only when tracking a real physiological trajectory. The top panel shows the controller output which is the applied pulse-width of the current pulses delivered at $20 \mathrm{~Hz}$ (with saturation limits set to 100 and $500 \mu \mathrm{s}$ ), the middle panel shows the desired (solid line) and actual (dashed line) normalized concentration of $\mathrm{Ca}$ ions (desired concentration is calculated by the sliding mode control law), and the bottom panel the reference (solid line) and actual (dashed line) knee joint angle trajectory. We can observe very good tracking performance.

Table 2 in turn shows the root-mean-square tracking error as a function of controller parameters $\lambda$ and $k$ when the knee extensors and knee flexors were stimulated with correct, nominal controller parameters.
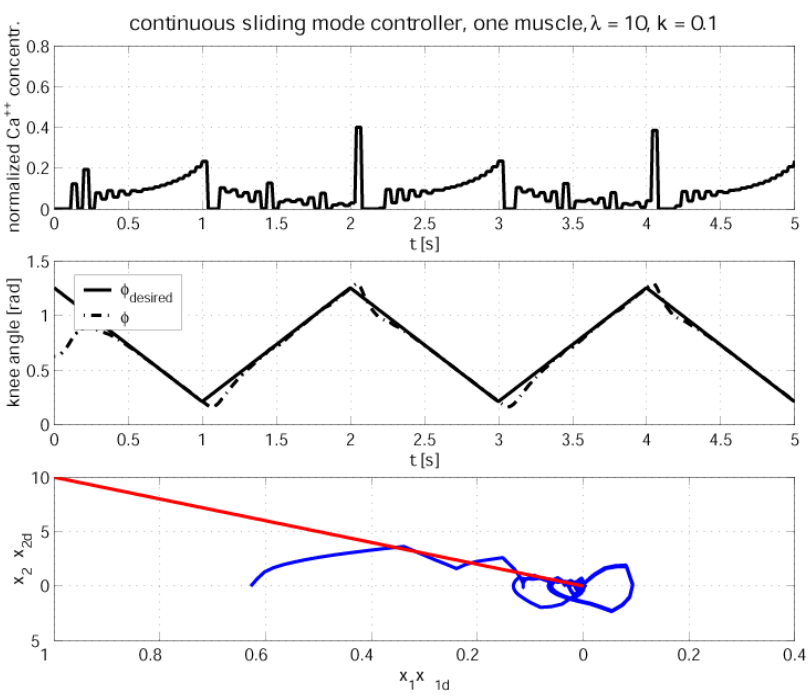

Fig.1: Continuous sliding mode controller for subsystem 1 - stimulation of the knee extensor muscle group only.

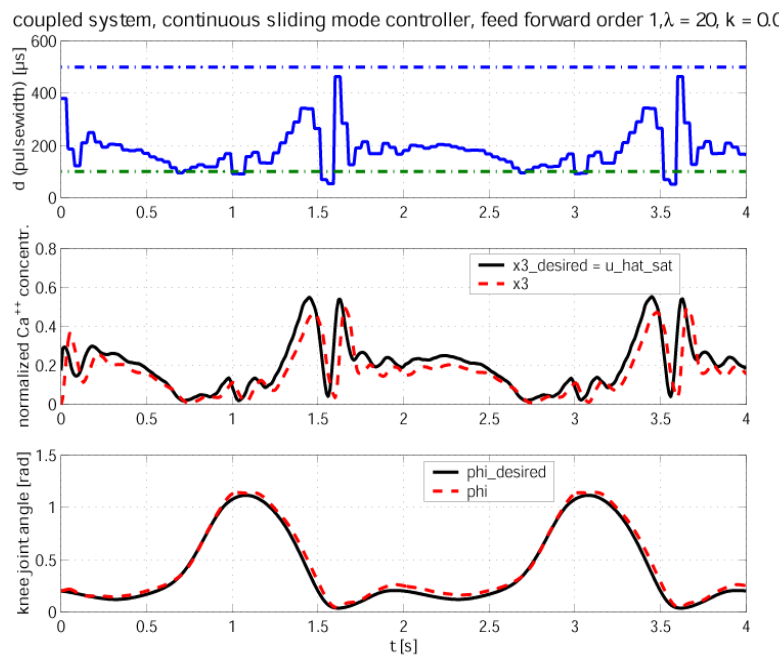

Fig.2: Continuous sliding mode controller for the combined system - stimulation of the knee extensor muscle group only.

Table 2: SM controller for subsystem 1 (two muscles)

\begin{tabular}{c|ccc}
\hline $\mathrm{k}$ & $\begin{array}{c}\mathrm{e}_{\text {RMS }}[\mathrm{deg}] \\
(\lambda=10)\end{array}$ & $\begin{array}{c}\mathrm{e}_{\text {RMS }}[\mathrm{deg}] \\
(\lambda=20)\end{array}$ & $\begin{array}{c}\mathrm{e}_{\text {RMS }}[\mathrm{deg}] \\
(\lambda=30)\end{array}$ \\
\hline 0.01 & 4.73 & 3.68 & 3.25 \\
0.05 & 2.88 & $\mathbf{2 . 6 3}$ & 2.98 \\
0.1 & 2.72 & 3.11 & 4.01 \\
0.2 & 3.57 & 4.65 & 5.83 \\
\hline
\end{tabular}

By stimulating an agonist/antagonist (flexor/extensor) muscle pair we have achieved slightly better tracking errors as with the stimulation of one muscle only (compare with Table 1).

\subsection{Robustness to parameter variations}

Each of the following control law terms - fit, $I_{t o t}, \tau_{g}$, and $\tau_{p}$ - was scaled with $0.5,0.8,1.2$, and 1.5 
respectively, and the simulations with ramp tracking were repeated. Next we have calculated the relative changes in the RMS tracking error and plotted them in polar plots. If the tracking error was reduced, the relative errors lied inside the unit circle, and if the error due to parameter variation increased, then the relative errors lied outside the unit circle. Figs. 3 and 4 show the results for $\lambda=20$ and $k=0.05$, and Fig.5 for $\lambda=20$ and $k=0.1$ (normalized to nominal RMS errors of 2.92, 2.63, and 4.77 degrees respectively). The values for overestimated model parameter-terms are plotted on the top-right axes (angles: 0, 45, 90, and 135 degrees), and the values for underestimated model parameter-terms on the bottom-left axes (angles: 180, 225, 270, and 315 degrees). The values connected with solid line represent variations of $20 \%$, i.e. 0.8 and 1.2 , and those connected with dashed line variations of $50 \%$, i.e. 0.5 and 1.5 .

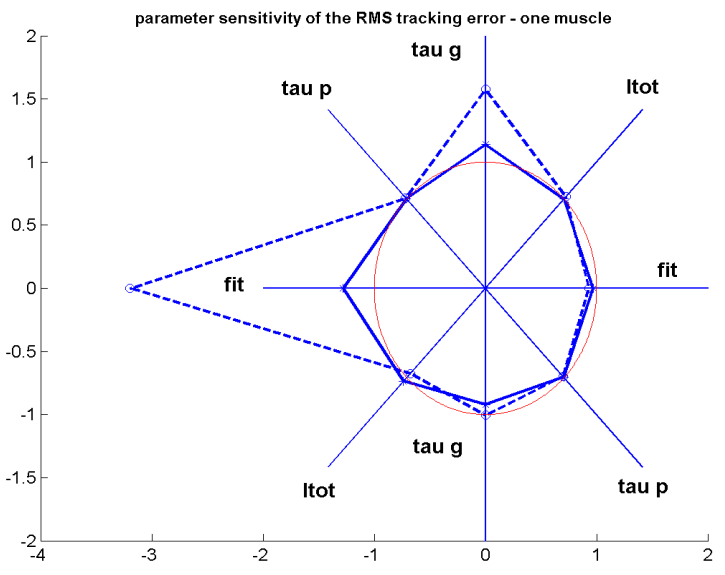

Fig.3: Polar plot showing the sensitivity of the RMS tracking error to different parameter-term variations in case of stimulation of one muscle and for $\mathrm{k}=0.05$.

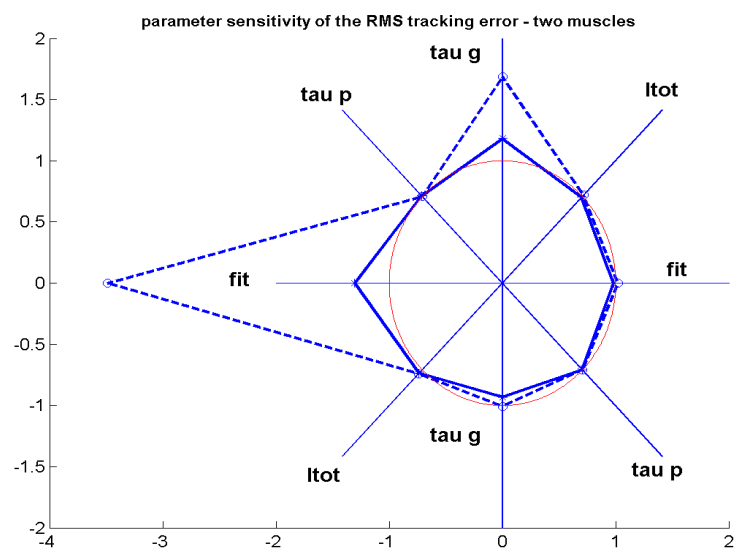

Fig.4: Polar plot showing the sensitivity of the RMS tracking error to different parameter-term variations in case of stimulation of two muscles and for $\mathrm{k}=0.05$.
In Figs. 3 and 4 we can observe that the sensitivity to parameter-term variations was similar in the case of one or two stimulated muscles. Largely increased errors were observed only in the case of overestimated $\tau_{g}$ term (1.5-fold RMS error) and underestimated fit term (more than 3-fold RMS error). Increasing the controller parameter $k$ to 0.1 resulted in increased overall RMS error, but the robustness to model parameter-term variations was increased (Fig.5). In fact, for most estimated model parameter-term variations the RMS error was reduced (except for underestimated fit term where we still observed a 1.5-fold increase in the RMS error). These results demonstrate that the fit term should rather be overestimated than underestimated.

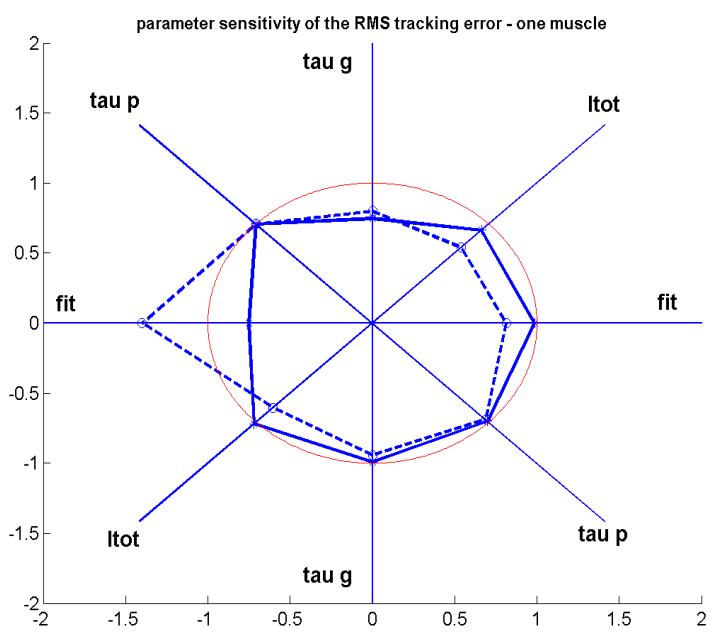

Fig.5: Polar plot showing the sensitivity of the RMS tracking error to different parameter-term variations in case of stimulation of one muscle and for $\mathrm{k}=0.1$.

\section{DISCUSSION}

The results of this computer simulation study are very promising. The developed sliding mode FES controller achieved good tracking performance with low root-mean-square tracking errors (2-3 degrees) at physiological walking speeds. Moreover, it never became unstable and was shown to be robust to model uncertainties. The controller is currently being tested in actual experiments. If similarly good performance will be achieved as in simulations, the controller will be augmented to control more than one degree of freedom (concurrent control of hip, knee, and ankle). At the end we would like to mention that a similar control problem was also tackled in (Chang, et al., 1997), but the controller design was not model-based. These authors have used a neural network-PID controller to control the knee joint angle. However, no proof of stability and robustness was given. On the other hand, the authors did report on experimental results with sinusoidal reference tracking. 


\section{REFERENCES}

Chang, G.C., Luh, J.J., Liao, G.D., Lai, J.S., Cheng, C.K., Kuo, B.L., and T.S. Kuo (1997). NeuroControl System for the Knee Joint Position Control with Quadriceps Stimulation. IEEE TRE, 5(1): 2-11.

Crago, P.E., Lan, N., Veltink, P.H., Abbas, J.J., and C. Kantor (1996). New control strategies for neuroprosthetic systems. J. of Reh. Res. and Dev., 33(2): 158-172.

Curk, B., Jezernik, K., Harnik, J., and A. Sabanovic (1996). Chattering reduction in VSC systems of robotic manipulators. In: Proceedings of $13^{\text {th }}$ IFAC World Congress on Automatic Control, San Francisco, USA, 503-508.

Jezernik, S. and R. Riener (1999). A computer simulation of tuned PID and continuous sliding mode FES control. In: Proceedings of the International Biomechatronics Workshop, Enschede, The Netherlands, 37-41.

Riener, R. and T. Fuhr (1998). Patient-driven control of FES supported standing-up: A simulation study. IEEE TRE, 6(2): 113-124.

Slotine, J.J.E. and J.A. Coetsee (1986). Adaptive sliding controller synthesis for nonlinear systems. Int. J.Control, 43(6): 1631-1651.

Utkin, V.U. (1992). Sliding Modes in Control and Optimization. Springer-Verlag, Berlin. 